Results Four SLE-risk genes were found to be associated with a decreased time until the first MI; PTPN22 (OR 1.61, $\mathrm{p}=0.041)$, NCF2 (OR 2.47, $\mathrm{p}=2.1 \times 10^{-3}$ ), STAT4 (OR 1.66, $\left.\mathrm{p}=5.2 \times 10^{-3}\right)$ and IL12A (OR 1.45, $\left.\mathrm{p}=0.047\right)$ and were included in a PRS. The PRS was associated with a higher cumulative prevalence of $\mathrm{MI}$ in both the discovery cohort $\left(p=1.1 \times 10^{-5}\right.$, fig $\left.1 \mathrm{~A}\right)$ and replication cohort $\left(\mathrm{p}=7.7 \times 10^{-3}\right.$, fig 1B). Exploring the PRS further in the replication cohort, patients in the high, compared to the low, PRS-quartile were more often male $\left(\mathrm{p}=1.3 \times 10^{-3}\right)$, and displayed higher prevalence of the ACR-1982 nephritis and immunological criteria $\left(\mathrm{p}=4.1 \times 10^{-4}\right.$ and $\left.\mathrm{p}=0.036\right)$ (fig1C).

Analyzing combinations of the identified SNPs, we found the prevalence of $\mathrm{MI}$ to be further increased in patients homozygous for both NCF2+STAT4 $\left(\mathrm{p}_{\text {discovery }}=1.6 \times 10^{-3}\right.$, $\mathrm{p}_{\text {re- }}$ plication $=0.015)$ or $S T A T 4+I L 12 A \quad\left(p_{\text {discovery }}=3.0 \times 10^{-5}\right.$, p preplication $=0.036$ ) (fig1D).

Conclusion A high polygenic risk score for MI in SLE is associated with an increased prevalence of myocardial infarction. If confirmed in prospective studies, our results suggest that genetic profiling may be useful for predicting MI in patients with SLE.

\section{P92 GENOMIC CONVERGENCE OF LOCUS-BASED GWAS META-ANALYSIS IDENTIFIES DDX11 AS A NOVEL SYSTEMIC LUPUS ERYTHEMATOSUS GENE}

${ }^{1}$ Mohammad Saeed, ${ }^{2}$ Alejandro Ibáñez-Costa, ${ }^{2}$ Alejandra María Patiño-Trives, ${ }^{2}$ María Ángeles Aguirre, ${ }^{2}$ Chary López-Pedrera. ${ }^{1} I m m u n o C u r e$, Karachi, Pakistan; ${ }^{2}$ Rheumatology Service, IMIBIC/Reina Sofia Hospital, University of Cordoba, Cordoba, Spain

10.1136/lupus-2020-eurolupus. 136

Background Systemic lupus erythematosus (SLE) is a complex autoimmune disorder with significant genetic underpinnings. Multiple genome-wide association studies (GWAS) have been conducted with identification of several SLE genes and loci. However, these only explain about $15 \%$ of SLE genetic risk indicating that additional loci of modest effect remain to be discovered. Association clustering methods such as OASIS are more apt than single-variant analysis for identifying modest genetic effects.

Methods Previously, OASIS analysis of two dbGAP GWAS datasets (6077 subjects; 0.75 million single-nucleotide polymorphisms (SNPs)) identified 30 SLE associated loci. In the present study, 410 genes were mapped to these loci and investigated for gene expression in 3 SLE GEO datasets. A fourth expression dataset, GSE50395, from Cordoba, Spain was used for validation. Expression quantitative trait loci (eQTL) analysis was performed using Blood eQTL browser for significant SNPs in loci harboring differentially expressed genes. Protein network analysis was performed using STRING to determine functional pathways involved. Confirmatory qPCR on monocytes of 12 healthy donors and 12 SLE patients was performed for the significant gene.

Results This genetic and functional analysis identified 187 genes in GEO datasets that were differentially expressed in SLE. Of these 55 genes were found to be differentially expressed in at least 2 GEO datasets with all probes directionally aligned. In the Cordoba dataset, 10 genes were found to have altered expression in SLE. DDX11 was significantly down regulated in both the GEO $(\mathrm{P}=3.60 \mathrm{E}-02)$ and the Cordoba $(P=8.02 \mathrm{E}-03)$ datasets. The most significant SNP, rs3741869 (GWAS $P=3.2 \mathrm{E}-05)$ in OASIS locus 19 (chromosome 12p11.21), containing the gene DDX11, was found to be a cis-eQTL regulating the expression of DDX11 $(\mathrm{P}=8.62 \mathrm{E}-05)$. DDX11 expression in SLE was confirmed to be down regulated using qPCR $(P=0.001)$, ratifying DDX11 as a novel gene associated with SLE. DDX11 interacted with multiple genes identified using genomic convergence of OASIS loci and expression analysis, including STAT1/STAT4. This study identified several novel SLE candidate genes as well, such as NRF1, TTC9 and BARHL1 which need additional data for verification.

Conclusion Genomic convergence approach with a locus-based test, OASIS, and expression studies on multiple datasets can help identify novel SLE genes. This study showed that DDX11, a RNA helicase thought to be involved in genome stability, is repressed in SLE and its expression is modulated by rs3741869.

\section{P93 ANALYSIS OF LOW-FREQUENCY AND RARE VARIANTS IN SWEDISH SLE PATIENTS WITH LUPUS NEPHRITIS}

Sule Yavuz. Uppsala University, Uppsala, Sweden

10.1136/lupus-2020-eurolupus. 137

Background Genome wide association studies (GWAS) have identified numerous SLE risk genes, however, few genes are reported as predisposing to the lupus nephritis (LN) phenotype. ${ }^{1}$

Objective To explore whether gene-based aggregates of low frequency $(\mathrm{MAF}<0.05)$ and/or rare $(\mathrm{MAF}<0.01)$ single nucleotide polymorphisms (SNPs) were associated with different subsets of LN or end stage renal disease (ESRD).

Methods We analyzed genotype data from Swedish SLE patients $(n=958)$. Data was generated by targeted sequencing of the coding and regulatory regions of 1900 genes involved in basic immune functions, inflammation and autoimmune diseases. The softwares GenePy ${ }^{2}$ and optimized sequence Kernel association test (SKAT-O) ${ }^{3}$ were used to analyze the impact of low frequency and rare variants in $\mathrm{LN}$ patients with different sub-phenotypes $(n=208)$ as compared to those $(n=621)$ who had not developed LN at the time of the study. We also performed logistic regression models with different sub phenotypes of LN as the outcome, adjusted for sex and age.

Results Two candidate genes; gelsolin (GSN; $5.97 \times 10^{-5}$ ) and Insulin like growth factor binding protein 7 were identified (IGFBP7; $5.95 \times 10^{-6}$ ) to have significantly higher GenePy scores in cases vs controls (bootstrap test, 1000 permutations). GSN, an actin-binding protein, is involved in non-AL renal amyloidosis and in the development of IgA nephropathy. GSN was associated with proteinuria (OR, [95\% CI]; 14.1 [2.966.9], $\mathrm{p}=0.001$ and ESRD (5.9[2.4-15.0], $\mathrm{p}=0.001)$ after adjusted for gender and age. IGFBP7, an emerging biomarker for acute kidney injury, was also significantly associated with Class3/4and ESRD $\left(p=4.9 \times 10^{-6}\right)$. SKAT-O identified one gene was associated with Class V; CTF1 (suggestive $\mathrm{p}<2.97 \times 10^{-5}$, Bonferroni corrected $p=0.05$ ).

Conclusion An aggregate association testing approach incorporating functional annotation revealed two putative risk loci (GSN and IGFBP7) associated with LN and ESRD. Further investigation needs to test their functional roles in detail.

\section{REFERENCES}

1. Niewold TB. Advances in lupus genetics. Curr Opin Rheumatol 2015 September;27(5):440-447. 